\title{
Spinal Cord Infarction and Patent Foramen Ovale: Is There a Link?
}

\author{
Marcelo Mendonça ${ }^{a, d} \quad$ Ana Sofia Correia ${ }^{a, d} \quad$ Ana Luís $^{b} \quad$ Pedro Soares $^{c}$ \\ Sofia Calado ${ }^{a}$ d Miguel Viana-Baptista ${ }^{a, d}$ \\ ${ }^{a}$ Neurology Department, ${ }^{b}$ Neurosurgery Department and ${ }^{c}$ Neuroimaging Department, \\ Hospital Egas Moniz - Centro Hospitalar de Lisboa Ocidental, and ${ }^{d}$ CEDOC, Faculdade de \\ Ciências Médicas da Universidade Nova de Lisboa, Lisbon, Portugal
}

\section{Key Words}

Spinal cord infarction · Stroke $\cdot$ Patent foramen ovale $\cdot$ Right-to-left shunting $\cdot$ Atrial septal aneurysm

\begin{abstract}
Spinal cord infarction (SCI) is an uncommon but important cause of acute myelopathy. Nevertheless, contrary to cerebral stroke, the discussion about paradoxical embolism as a cause of cryptogenic SCI remains dubious. We describe the case of a 24-year-old woman who developed sudden-onset back pain followed by upper limb paralysis. T2-weighted MRI demonstrated hyperintense signal, extending from C5 to D1 with corresponding restricted diffusion on diffusion-weighted MRI and reduction of the apparent diffusion coefficient. Diagnostic workup, including lumbar puncture, showed no changes. Transcranial Doppler showed a right-to-left shunt with an uncountable number of microembolic signals after Valsalva maneuvers, and a patent foramen ovale (PFO) with an atrial septum aneurysm was identified. We discuss the paucity of evidence of right-to-left shunting in spinal diseases compared to cerebral events and the potential role of paradoxical embolism through PFO as a possible mechanism of $\mathrm{SCI}$.

(c) 2014 S. Karger AG, Basel
\end{abstract}

\section{Introduction}

Spinal cord infarction (SCI) is a rare but important cause of acute myelopathy, accounting for 5-8\% of all cases [1]. Differential diagnosis, mainly with acute inflammatory diseases, is mandatory, and diffusion-weighted imaging (DWI) MRI - although seldom performed due to technical issues - has been described as a useful tool in this distinction [2]. The most common cause of SCI is aortic disease; nevertheless, a definite cause is not found in $60-75 \%$

Miguel Viana-Baptista, MD, PhD

Serviço de Neurologia

Hospital Egas Moniz - Centro Hospitalar de Lisboa Ocidental

Rua da Junqueira 126, PT-1349-019 Lisbon (Portugal)

E-Mail mvianabaptista@fcm.unl.pt 
of the cases, which is a higher rate than that found in brain infarction $[3,4]$. The presence of a patent foramen ovale (PFO) with atrial septum aneurysm (ASA) has been described as a risk factor for cryptogenic brain infarction. This is not clearly established for SCI, as there are only 3 reported cases of SCI associated with a PFO [5-7]. However, right-to-left shunting has also been related to spinal infectious diseases by septic embolism [8-10]. We describe the case of a SCI associated with a PFO with a borderline ASA and discuss the possible role of right-to-left shunting on cryptogenic spinal infarction.

\section{Case Report}

A 24-year-old female patient, under oral contraceptive treatment with 0.015 mg ethinylestradiol and $0.006 \mathrm{mg}$ gestodene, woke up in the middle of the night with sudden, intense and self-limited interscapular pain and paresthesias in both hands. She went back to sleep and, when she woke up again a few hours later, she noticed weakness in both hands and abdominal wall numbness. The patient denied urinary or other sphincter dysfunction. She had no relevant medical, surgical or family history and denied alcohol, tobacco or other drug use or having suffered a relevant trauma in the previous days. On admission, her blood pressure was 132/90 mm Hg. The general examination was unremarkable, and neurological examination disclosed flaccid distal paresis of the upper limbs with decreased osteotendinous reflexes in the upper limbs and abnormal plantar responses. Pain sensation was diminished between D2 and L2 dermatomes. There was no weakness of the lower limbs. MRI of the cervical and dorsal spine showed T2-weighted sequence change in the signal between C5 and D1 at the levels of the anterior horns of the spinal grey matter, which was more evident on the left where they also involved the anterolateral white matter and the lateral cord (fig. 1a, b) with restricted diffusion on DWI (fig. 1c, d), highly suggestive of SCI. Brain MRI showed no changes. A diagnostic workup with complete blood count, biochemical testing, erythrocyte sedimentation rate, diabetes screening, cerebrospinal fluid examination, immunological study, including complement levels, anti-nuclear antibodies and antiphospholipid antibodies and infections, with syphilis screening and HIV, was negative. Abnormalities of the spinal cord vascular system (namely arteriovenous malformations) were excluded on the ground of normal-selective digital subtraction angiographic findings. Cervical vessel ultrasound disclosed neither carotid nor vertebral stenosis nor atherosclerotic changes. Transcranial Doppler showed patency of major cerebral arteries, and saline IV infusion detected a right-to-left at-rest grade III shunt according to the Spencer grading scale (16-50 microembolic signals) [11], with an uncountable number of microembolic signals (shower effect, grade $\mathrm{V}+$ ) after a Valsalva maneuver. Transesophageal echocardiogram showed a PFO and an interatrial septum protruding to the right atrium $10 \mathrm{~mm}$ beyond the original position of the interatrial septum. A 24-hour Holter ECG did not detect any potentially embolic arrhythmias.

In the absence of other causes, the patient was treated with oral anticoagulation therapy until a percutaneous PFO closure was performed with an Amplatzer device. The patient was subsequently started on 100-mg aspirin treatment. One year after the primary event, the patient scored 2 on the modified Rankin Scale with a forearm paralysis. No evidence of shunting was seen on the transesophageal echocardiogram. 
Mendonça et al.: Spinal Cord Infarction and Patent Foramen Ovale: Is There a Link?

\section{Discussion}

Right-to-left shunts have been discussed as relevant causes of cryptogenic stroke [12]. The role of PFO in stroke, mainly when associated with ASA, is well described [13]. Unfortunately, however, there are no systematic studies of its prevalence in or impact on cryptogenic SCI.

PFO has also been linked with cryptogenic brain abscess, and some studies have shown the co-occurrence of PFO with intramedullary abscesses of the spinal cord (IASC) [8, 9]. IASC is a rare condition that usually occurs secondary to bacteremia from cardiac, pulmonary or urinary foci. Hoche demonstrated that the introduction of micro-organisms into the circulation of experimental animals did not result in the development of ISCA; however, if both microorganisms and thrombi were introduced, ISCA developed [9]. Therefore, spinal embolism is understood as a relevant disease mechanism. Higuchi et al. [8] and Terterov et al. [9] identified Streptococcus viridians as the causative agent of IASC. This is a well-known oral low-pathogenicity agent that can cause transient asymptomatic bacteremia in healthy patients. In their case report, Terterov et al. [9] suggested that this transient bacteremia infected a subclinical deep vein thrombosis and the paradoxical embolism was facilitated by the patient PFO. The right-to-left shunting would allow pathogens to bypass pulmonary capillary filtering and enter arterial circulation easier. Besides these reports, arteriovenous pulmonary malformations or atrial septal defects have been reported in patients with IASC $[8,10]$. The co-occurrence of PFO and IASC makes the hypothesis of the mechanism of paradoxical infections through PFO plausible. We propose that the same rationale could be considered for $\mathrm{SCI}$, and that paradoxical embolism through a PFO can be a possible cause of $\mathrm{SCI}$ in the presence of an associated ASA.

As the onset of symptoms in our patient happened during her sleep, no Valsalva maneuver was detected. Nevertheless, while sleeping, there can be changes of intrathoracic pressures that can facilitate a transient rise in atrial pressure and co-occurring embolism [14]. Simultaneously, we have evidence that the Valsalva maneuver significantly increased the number of microembolic signals detected by transcranial Doppler; thus, we admit the possibility that a Valsalva-like mechanism could have a role in the paradoxical embolism. Due to its specific vascular anatomy, the spinal cord is a rarer site for an embolism than the brain. The rarity of this event, facing the high frequency of PFO and ASA in the normal population, probably explains the difficulty of establishing the role of PFO or PFO with ASA in spinal vascular disorders.

We propose that the search for a right-to-left shunt is reasonable in the presence of a SCI with an unknown cause, and, although the role of PFO is not clearly established in the etiology and long-term follow-up, its closure can be considered, probably in the same way as in cerebral stroke.

\section{Conclusion}

Based on the current evidence, it is impossible to establish a definite association between PFO, a frequent condition, and SCI, a rare disorder. Facing the lack of studies on the management of infarction in the presence of PFO or even SCI, we considered closing the PFO a safe and effective approach in our case, as we reckoned that the recurrence of embolic events might be significant in this young patient. With this report, we try to fundament the role of PFO as a potential cause to be considered in cryptogenic SCI. 
The relative rarity of SCI and the difficulties in its diagnosis make research in this area difficult. With this report, we expect to have highlighted the need to understand the role of right-to-left shunts in spinal disorders and to motivate further studies. The creation of a multinational SCI registry would probably be a helpful effort to understand the SCI epidemiology and pathology and to optimize its management.

\section{Disclosure Statement}

The authors have no conflicts of interest to declare.

\section{References}

$\checkmark 1$ Nedeltchev K, Loher T], Stepper F, et al: Long-term outcome of acute spinal cord ischemia syndrome. Stroke 2004;35:560-565.

2 Nogueira RG, Ferreira R, Grant PE, Maier SE, Koroshetz WJ, Gonzalez RG, Sheth KN: Restricted diffusion in spinal cord infarction demonstrated by magnetic resonance line scan diffusion imaging. Stroke 2012;43:532-535

3 Cheng MY, Lyu RK, Chang YJ, Chen RS, Huang CC, Wu T, Lee TH, Lu CS, Ro LS: Spinal cord infarction in Chinese patients. Clinical features, risk factors, imaging and prognosis. Cerebrovasc Dis 2008;26:502-508.

-4 Novy J, Carruzzo A, Maeder P, Bogousslavsky J: Spinal cord ischemia: clinical and imaging patterns, pathogenesis, and outcomes in 27 patients. Arch Neurol 2006;63:1113-1120.

-5 Petruzzellis M, Fraddosio A, Giorelli M, et al: Posterior spinal artery infarct due to patent foramen ovale: a case report. Spine (Phila Pa 1976) 2010;35:E155-E158.

-6 Mori S, Sadoshima S, Tagawa K, et al: Massive spinal cord infarction with multiple paradoxical embolism: a case report. Angiology 1993;44:251-256.

7 Domingues RB, Vilas-Novas MF: Cervical spinal cord infarct associated with patent foramen ovale. Arq Neuropsiquiatr 2012;70:381-382.

-8 Higuchi K, Ishihara H, Okuda S, Kanda F: A 51-year-old man with intramedullary spinal cord abscess having a patent foramen ovale. BMJ Case Rep 2011;2011:bcr1120103512.

$>9$ Terterov S, Taghva A, Khalessi AA, Kim PE, Liu C: Intramedullary abscess of the spinal cord in the setting of patent foramen ovale. World Neurosurg 2011;76:361.e11-e14.

10 David C, Brasme L, Peruzzi P, Bertault R, Vinsonneau M, Ingrand D: Intramedullary abscess of the spinal cord in a patient with a right-to-left shunt: case report. Clin Infect Dis 1997;24:89-90.

11 Van H, Poommipanit P, Shalaby M, Gevorgyan R, Tseng CH, Tobis J: Sensitivity of transcranial Doppler versus intracardiac echocardiography in the detection of right-to-left shunt. JACC Cardiovasc Imaging 2010;3:343-348.

12 Piechowski-Jozwiak B, Bogousslavsky J: Stroke and patent foramen ovale in young individuals. Eur Neurol 2013;69:108-117.

13 Mas JL, Arquizan C, Lamy C, Zuber M, Cabanes L, Derumeaux G, Coste J; Patent Foramen Ovale and Atrial Septal Aneurysm Study Group: Recurrent cerebrovascular events associated with patent foramen ovale, atrial septal aneurysm, or both. N Engl J Med 2001;345:1740-1746.

14 Arboix A, Alió J: Cardioembolic stroke: clinical features, specific cardiac disorders and prognosis. Curr Cardiol Rev 2010;6:150-161. 

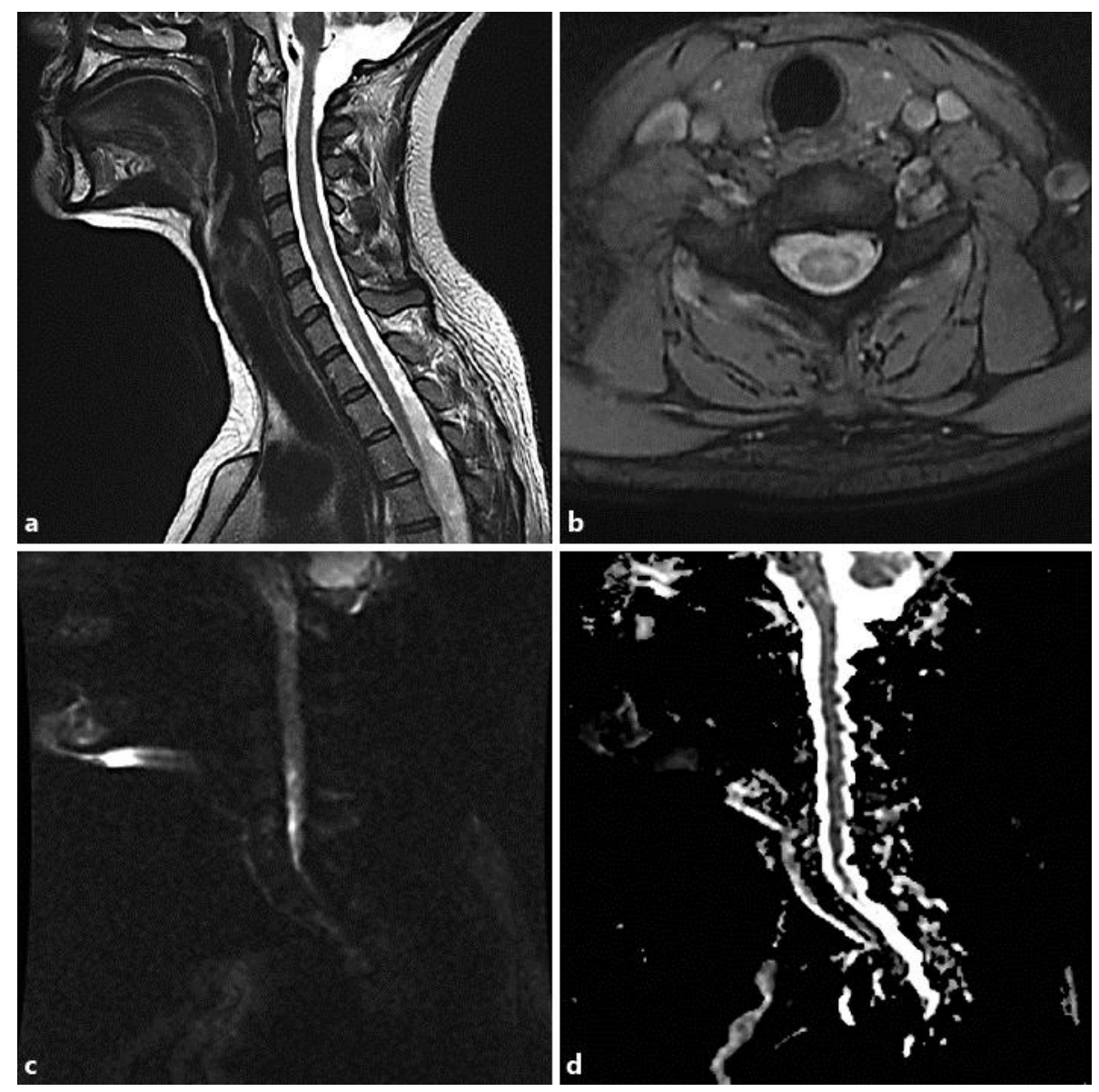

Fig. 1. a T2-weighted sagittal MRI of the cervical spine. b T2-weighted axial MRI of the cervical spine. c DWI sequence. $\mathbf{d}$ ADC map. Lesion with hyperintense signal, extending from C5 to D1 with slight edema (a). The lesion involves the anterior horns of the spinal grey matter (b), and there is diffusion restriction from C5 to C7 (c, d), corresponding to the lesion depicted in a. 